\title{
Toward Ultrafast Electron Microscope with Femtosecond Temporal Resolution, Atomic-Level Spatial Resolution, and Single-Shot Imaging Capability
}

\author{
Katsumi Tanimura
}

\author{
The institute of Scientific and Industrial Research, Osaka University, Osaka, Japan
}

Innovative progress in materials science in the last century made it possible to determine the structures of matters at the atomic-level $(1 \AA)$ resolution, and to detect ultrafast structural dynamics in the temporal domains as short as $10^{-13} \mathrm{~s}$. However, one important limitation was left unsolved; that the ultimate spatial and temporal resolutions are incommensurate. At the beginning of this century, the new innovation has started to realize direct structural determination of materials in ultrafast processes. In particular, ultrafast time-resolved electron diffraction methods have developed dramatically to capture structural dynamics in short temporal domains, as reviewed in Refs. 1 and 2. The next milestone of ultrafast structural dynamic studies may be to establish the femtosecond (fs) time- resolved electron microscope (U-TEM) with atomic-level spatial resolution and with a single-shot imaging capability [3]. Here I describe the present status of our study to construct such a U-TEM, based on our single-shot fs electron diffractometer using relativistic ultrafast electron pulses.

The relativistic electron pulses give two crucial advantages over conventional DC-accelerated electron beams in electron diffraction and electron microscope. First, $\mathrm{MeV}$ electrons greatly enhance extinction distance for elastic scattering, and provide structural information almost free from any multiple diffraction effects; the kinematic theory assuming single scattering events can be applied. This feature allows us to obtain ultrafast atomistic-scale structural evolution by combining time-resolved diffraction results with state-of-art theoretical modeling, which is capable of quantitatively reproducing the time evolution of diffraction intensities. Detailed atomistic evolutions have been obtained in laser-generated warm-dense gold [4]. This is a version of electron diffractive imaging.

Second, for relativistic electron beams minimal longitudinal pulse broadening will result, even with more than $10^{9} \mathrm{e} / \mathrm{cm}^{2}$ per pulse. This enables us to perform single-shot transmission diffraction at fstemporal resolution, crucial for irreversible processes such as laser-induced solid/liquid transitions. More importantly, this feature opens a possibility to obtain single-shot imaging of atomic structures, by combining appropriate lens systems and high-sensitive detectors. For any atomic motions in fs-temporal domains, single-shot imaging is crucial as we study structural changes beyond ergodic picture.

The renovation from the electron diffractometer to microscope needs several challenging developments. These include ultimate stability and monochromaticity of energies of $\mathrm{MeV}$ electrons generated by $\mathrm{rf}$ photocathode, precise beam control for collimation, focusing and expansion, and high-sensitive detection for imaging. The present status of these developments is discussed in details.

\section{References:}

[1] A. H. Zewail, Annu. Rev. Phys. Chem. 57, 65 (2006).

[2] G. Sciainiland R. J. Dwayne Miller, Rep. Prog. Phys. 74, 096101 (2011).

[3] D. J. Flannigan and A. H. Zewail, Accounts Chem. Res. 45, 1828 (2012).

[4] S. L. Daraszewicz, et al., Phys. Rev. B 88, 184101 (2013). 
